\title{
The Endurance and Selectivity of Spatial Patterns of Long-Term Potentiation/Depression in Dendrites under Homeostatic Synaptic Plasticity
}

\author{
Ithai Rabinowitch and Idan Segev \\ Interdisciplinary Center for Neural Computation and the Department of Neurobiology, Institute of Life Sciences, The Hebrew University of Jerusalem, \\ Edmond Safra Campus, Givat Ram, Jerusalem, 91904, Israel
}

\begin{abstract}
We investigated analytically and numerically the interplay between two opposing forms of synaptic plasticity: positive-feedback, longterm potentiation/depression (LTP/LTD), and negative-feedback, homeostatic synaptic plasticity (HSP). A detailed model of a CA1 pyramidal neuron, with numerous HSP-modifiable dendritic synapses, demonstrates that HSP may have an important role in selecting which spatial patterns of LTP/LTD are to last. Several measures are developed for predicting the net residual potentiation/depression after HSP from the initial spatial pattern of LTP/LTD. Under a local dendritic HSP mechanism, sparse patterns of LTP/LTD, which we show, using information theoretical tools, to have a significant impact on the output of the postsynaptic neuron, will persist. In contrast, spatially clustered patterns with a smaller impact on the output will diminish. A global somatic HSP mechanism, conversely, will favor distally occurring LTP/LTDs over proximal ones. Despite the negative-feedback nature of HSP, under both local and global HSP, numerous synaptic potentiations/depressions can persist. These experimentally testable results imply that HSP could be significantly involved in shaping the spatial distribution of synaptic weights in the dendrites and not just normalizing it, as is currently believed.
\end{abstract}

Key words: homeostatic plasticity; LTP; long-term potentiation; synaptic scaling; compartmental model; CA1 pyramidal; dendrite

\section{Introduction}

Long-term potentiation and depression (LTP/LTD) (Bliss et al., 2004) are probably the most studied forms of synaptic plasticity, receiving a lot of attention for their relatively fast induction rate and their persistence over long periods of time (hours, days, and even longer). However, synapses that are subject to positivefeedback LTP/LTD also undergo simultaneous but much slower negative-feedback modifications termed homeostatic synaptic plasticity (HSP) (Davis and Bezprozvanny, 2001; Turrigiano and Nelson, 2004), which seem to compensate for persisting deviations of activity from a physiologically and functionally desired level. What is the net outcome of these two presumably opposing plasticity mechanisms?

Several studies have addressed this question (van Rossum et al., 2000; Kempter et al., 2001; Yeung et al., 2004; Toyoizumi et al., 2005), but they were all performed on "point-neuron" models lacking a spatial dimension. Such models cannot address the as yet unresolved issue of whether HSP acts locally, sensing the level of activity at the site of each synapse and accordingly modifying the strength of each individual synapse or, globally, monitoring

Received April 5, 2005; revised Nov. 5, 2006; accepted Nov. 6, 2006.

This work was supported by a grant from the Edmond J. Safra foundation, National Institutes of Health Grant IR01-MH59976-01A2, and Israeli Science Foundation Grant 10/256. I.R. is supported by the Horowitz fund fellowship. We thank Mickey London for help with the synapse information efficacy measure.

Correspondence should be addressed to Idan Segev, Interdisciplinary Center for Neural Computation and the Department of Neurobiology, Institute of Life Sciences, The Hebrew University of Jerusalem, Edmond Safra Campus, Givat Ram, Jerusalem, 91904, Israel. E-mail: idan@lobster.Is.huji.ac.il.

D01:10.1523/JNEUROSCI.4333-06.2006

Copyright $\odot 2006$ Society for Neuroscience $\quad$ 0270-6474/06/2613474-11\$15.00/0 activity at the level of the entire cell (e.g., average firing rate) and modifying all synaptic weights in unison. Although most of the literature assumes HSP to be driven by a global mechanism scaling synaptic strength (Turrigiano and Nelson, 2004), recent evidence seems to favor a local one (Ju et al., 2004; Sutton et al., 2004, 2006; Thiagarajan et al., 2005). To address how this may affect the interplay between HSP and LTP/LTD, we compared between the two.

We combine in this work an analytical study with numerical simulations of a detailed reconstructed model of a CA1 pyramidal neuron invested with numerous HSP-modifiable excitatory synapses that change according to either the local (l-HSP) or global ( $g$-HSP) level of activity. Because HSP has been shown experimentally to be linked to changes in membrane potential (Leslie et al., 2001; Paradis et al., 2001; Burrone et al., 2002; Moulder et al., 2003) and because the spread of membrane potential in dendrites is currently far better understood than the complex dynamics of calcium or any other biochemical agent, we chose to investigate a voltage-based model of HSP. We nevertheless maintain that our conclusions generalize to alternative HSP mechanisms.

We found that the amount of potentiation remaining after HSP can be determined by the initial spatial pattern of LTP/LTD. $g$-HSP will tend to preserve spatial patterns that have less potentiated synapses at proximal dendritic sites and more potentiated synapses at distal locations. In contrast, $l$-HSP will tend to favor (preserve) sparse patterns of LTP/LTD over dense ones. We show using the synapse information efficacy (SIE) measure (London et al., 2002) that such sparse patterns have a substantially stronger impact on the output of a neuron. We conclude that HSP oper- 
A



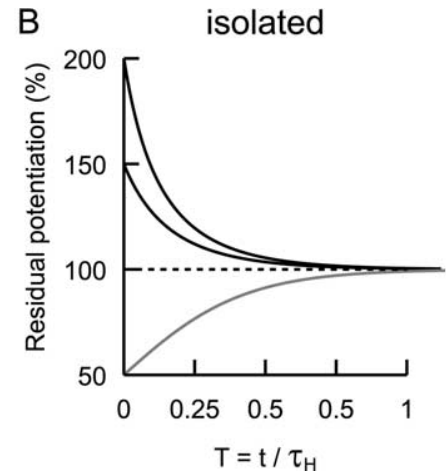

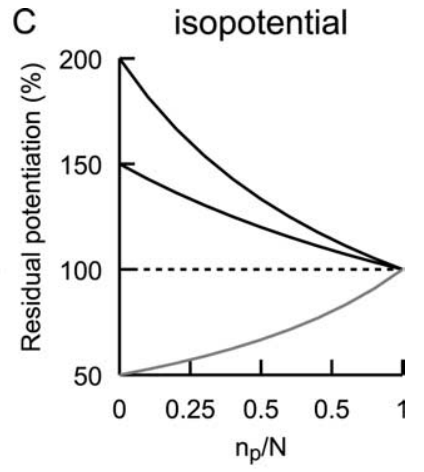

Figure 1. The amount of residual potentiation after HSP depends on the electrical properties of the dendrites and on the relative number of potentiated synapses. $\boldsymbol{A}$, Schematic drawings of three hypothetical cases for the electrical properties of a dendritic branch: complete electrical isolation (top), the isopotential case (middle), and an electrically distributed dendrite (bottom) with longitudinal current spread. $B$, Decrease of the residual potentiation with time (in units of the HSP time constant, $\tau_{H}$ ) attributable to HSP according to Equation A7 (supplemental Appendix I, available at www.jneurosci.org as supplemental material) in the extreme isolated case for initial potentiations of 150 and 200\% (black) and an initial depression of 50\% (gray). C, Steadystate levels of the residual potentiation attributable to HSP in the isopotential case as a function of the ratio between the number of potentiated synapses, $n_{p}$, and the total number of synapses, $N$, for initial potentiations of 150 and $200 \%$ (black) and an initial depression of $50 \%$ (gray).

ating in an electrically distributed dendritic tree has an active role in deciding the fate of LTP/LTD at each synapse, and this fate strongly depends on the spatial context of LTP/LTD and on the nature of the HSP mechanism, local, global, or a combination of both.

\section{Materials and Methods}

Compartmental model. All simulations were designed and run using NEURON 5.8 (http://www.neuron.yale.edu). A reconstructed hippocampal CA1 pyramidal cell (Fig. $1 D$; kindly provided by N. Spruston, Northwestern University, Evanston, IL) equipped with a simple cylindrical axon was used as an electrically distributed neuron model. Passive properties were as follows: intracellular resistivity, $R_{i}=150 \Omega \cdot \mathrm{cm}$ in dendrites and $50 \Omega \cdot \mathrm{cm}$ in the axon; membrane resistance, $R_{\mathrm{m}}=28$ $\Omega \cdot \mathrm{cm}^{2}$; membrane capacitance, $C_{\mathrm{m}}=1 \mu \mathrm{F} / \mathrm{cm}^{2}$; resting potential, $V_{\text {rest }}=-65 \mathrm{mV}$. Dendritic spines were accounted for by a twofold increase in $C_{\mathrm{m}}$ and a corresponding decrease in $R_{\mathrm{m}}$. Unless otherwise mentioned, dendritic sections were divided into discrete $\Delta x=20 \mu \mathrm{m}$ long segments, and the integration time step was $\Delta t=25 \mu \mathrm{s}$.

Voltage-dependent currents. Voltage-dependent fast $\mathrm{Na}^{+}\left(I_{\mathrm{Na}}\right)$, delayed rectifier $\mathrm{K}^{+}\left(I_{\mathrm{K}}\right)$, and A-type $\mathrm{K}^{+}\left(I_{\mathrm{A}}\right)$ currents (Migliore et al., 1999 ) were incorporated in the neuron, with the following minor modifications. Only the proximal variant of $I_{\mathrm{A}}$ was used (Migliore et al., 1999); the additional inactivation gate for $I_{\mathrm{Na}}$ (Migliore, 1996) was not used; and $V_{1 / 2}$ for activation of $I_{\mathrm{Na}}$ in the axon was raised from -30 to $-25 \mathrm{mV} . I_{\mathrm{Na}}, I_{\mathrm{K}}$, and $I_{\mathrm{A}}$ channel densities in the dendrites were 60,50 , and $100+1.22 \cdot x \mathrm{pS} / \mu \mathrm{m}^{2}$, respectively, where $x$ indicates path distance from the soma in micrometers. Channel densities in the soma were identical to those in the adjoining dendritic branches. $I_{\mathrm{Na}}, I_{\mathrm{K}}$, and $I_{\mathrm{A}}$ channel densities in the axon were 35,000, 500 and $4000 \mathrm{pS} / \mu \mathrm{m}^{2}$, respectively.

Synapses and HSP. Excitatory fast synaptic conductance was modeled as a double exponential (the built-in NEURON point process Exp2Syn was used) with $\tau_{\text {rise }}=0.3 \mathrm{~ms}, \tau_{\text {decay }}=3 \mathrm{~ms}$, and reversal potential $E_{\text {syn }}=$ 0 . Unless otherwise mentioned, each synapse was randomly activated according to a Poisson distribution with a mean rate of $2 \mathrm{~Hz}$. The time constant for the HSP model, $\tau_{\mathrm{H}}$ (Eqs. 4, 5) was set to $100 \mathrm{~s}$; larger $\tau_{\mathrm{H}}$ values only increased the simulation time without changing the results. In Figures 2-4 and 6, $B$ and $C$, one model synapse was included in each one of 587 dendritic segments. In Figures 5 and 6 (except for Fig. $6 B, C$ ) the 50 longest end branches of the dendritic tree were divided into $\Delta x=$ $5 \mu \mathrm{m}$ long segments and the rest of the tree into $\Delta x=25 \mu \mathrm{m}$ long segments. With one synapse per segment, the total number of synapses was 1514. Simulations were run for $t_{1}=500 \mathrm{~s}$ simulation time. In Figures $2 G, 3 D$, and $4 B, t_{\mathrm{H}}=20 \mathrm{~s}$ (the time to reach steady state).
The SIE. To quantify the efficacy of potentiated synapses within different spatial patterns (see Fig. 4) not only in terms of their peak conductances but also in their ability to drive neuronal output, we used the SIE measure (London et al., 2002). Full details about this measure and its properties have been given previously (London et al., 2002). In brief, the SIE estimates the mutual information between presynaptic and postsynaptic spike trains by computing the difference between the estimated output entropy and the estimated conditional entropy of the output given the input. To overcome the entropy estimation bias attributable to the relatively weak contribution of individual synapses in the context of many other active synapses, we corrected the SIE by subtracting from the SIE raw value the difference between the output entropy and the conditional entropy of the output given a shuffled input train. For each SIE estimation, we averaged 25 such corrected SIE values, obtaining SDs at least one order of magnitude smaller than the average. This average was then used as the estimated SIE presented in Figure 4. All input and output spike trains were $1000 \mathrm{~s}$ long (enough for the SIE measure to converge). Bin size was $2 \mathrm{~ms}$, and the window was $15 \mathrm{~ms}$ long.

\section{Results}

\section{The residual potentiation after HSP in an isopotential neuron model}

Long-term potentiation (and diametrically, long-term depression) of excitatory synapses is expected to increase the average activity of the postsynaptic neuron. If persisting for a long period of time, this increased activity will be counterbalanced by regulatory mechanisms such as HSP that will decrease synaptic strength, so that the activity of the neuron can resume its target level. Therefore, after HSP, at least some of the initial synaptic potentiation, $p^{0}$, is expected to be lost, leaving what we shall call a residual potentiation, $\mathrm{rp}$, the net increase in the original synaptic strength resulting from the joint effects of LTP and HSP. What will be the size of the residual potentiation of a synapse after HSP (in what follows, we mostly refer to synaptic potentiation, but our conclusions symmetrically hold for synaptic depression)?

Several insights on this question may be gained by contemplating a very simple model. Consider a dendritic branch receiving $N$ synaptic inputs. On the one extreme, this branch could be divided into $N$ electrically decoupled isolated compartments such as illustrated in Figure $1 A$ (top). On the other extreme, this branch may be effectively isopotential (Fig. $1 A$, middle). Normally, the cable properties of dendritic branches lie somewhere between these two extremes, so that there will be current flow (or, e.g., a gradient of calcium concentration) along the dendritic cable, and membrane potential will vary between different synaptic locations (Fig. 1A, bottom). With a few simplifying assumptions, the amount of residual potentiation, rp, can be readily analyzed for the two extreme cases: complete electrical isolation and the isopotential case. At the heart of this analysis is a simple model for the HSP process that describes the very slow change of the peak synaptic conductance, $G_{\text {syn }, i}$ of each synapse, $i$, according to the difference between membrane potential, $V$, and a target membrane potential, $V_{\mathrm{trg}}$ :

$$
\tau_{\mathrm{H}} \cdot \frac{d G_{\text {syn }, i}}{d t}=\frac{V_{\mathrm{trg}}-V}{\kappa} \cdot G_{\mathrm{syn}, i},
$$


where $\tau_{\mathrm{H}}$ is the time constant for the HSP process, which is very large relative to the membrane time constant $\tau_{\mathrm{m}}$, and $\kappa=1$, in voltage units, maintains $G_{\text {syn }, i}$ in units of conductance. The solution to this equation for the isolated case (Fig. 1A, top) (supplemental Appendix I, Equation A7, available at www.jneurosci.org as supplemental material) is shown in Figure $1 B$, where $\operatorname{rp}(T)$, the amount of residual potentiation as a function of time (in units of $\left.\tau_{\mathrm{H}} ; T=t / \tau_{\mathrm{H}}\right)$ is plotted for initial potentiations $p^{0}=150$ and $200 \%$, as well as for a $50 \%$ initial depression of a synapse on the branch. Obviously, in this case, any potentiation is bound to decay to nil at the HSP timescale (of hours to days), eventually leaving no residual potentiation.

Conversely, in the case of an isopotential dendrite (Fig. 1A, middle), the HSP dynamics will multiplicatively scale down all synaptic weights by a global scaling factor, SF (supplemental Appendix I, available at www.jneurosci.org as supplemental material). As a consequence, in addition to the potentiated synapses, the other nonpotentiated synapses will also be weakened, thus supporting the potentiated synapses so that the latter will not necessarily lose all of their initial potentiation. How much of the initial potentiation will be rescued is a function of the number of potentiated synapses, $n_{p}$, relative to the total number of synapses, $N$. As an example, we have derived this function for the case in which all $n_{p}$ synapses are initially potentiated to the same level, $p^{0}$ (supplemental Appendix I, Equation A10, available at www.jneurosci.org as supplemental material). This is plotted in Figure $1 C$ (for $p^{0}=150,200$, and 50\%, as in Fig. $1 B)$. In the case in which the number of potentiated synapses is very small relative to the total number of synapses $\left(n_{p} / N \rightarrow 0\right.$ in Fig. 1C), these synapses will maintain their initial potentiations almost fully (rp will approach $p^{0}$ ). In contrast, as the number of potentiated synapses approaches the total number of synapses $\left(n_{p} / N \rightarrow 1\right.$ in Fig. $1 C$ ), the potentiation will be entirely lost ( $\mathrm{rp}$ will approach $100 \%)$. In fact, this is what happens in the isolated case where $n_{p}=N=1$.

\section{HSP in a detailed neuron model}

What would be the residual potentiation of synapses in a more realistic, electrically distributed dendritic tree? It can be expected to lie somewhere between the isopotential residual potentiation (Fig. 1C) and the complete abolishment of the initial potentiation, in the isolated case (Fig. $1 B$ ). As in the isopotential case, nonpotentiated synapses can help support the persisting potentiation of synapses, but the location (spatial distribution) of the participating synapses along the dendrite will now become important. To investigate this under much more realistic condi-
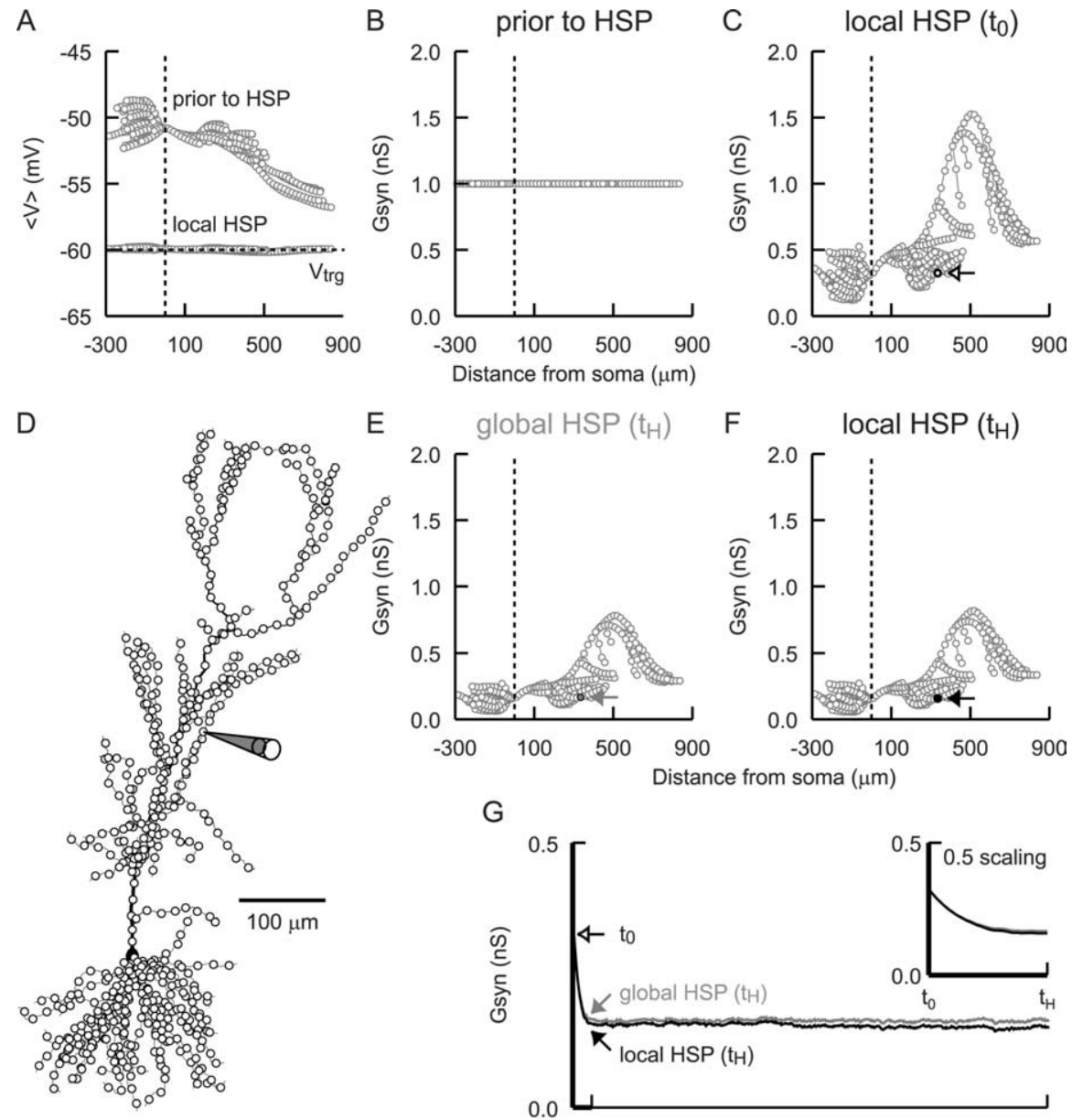

E global HSP $\left(t_{H}\right)$
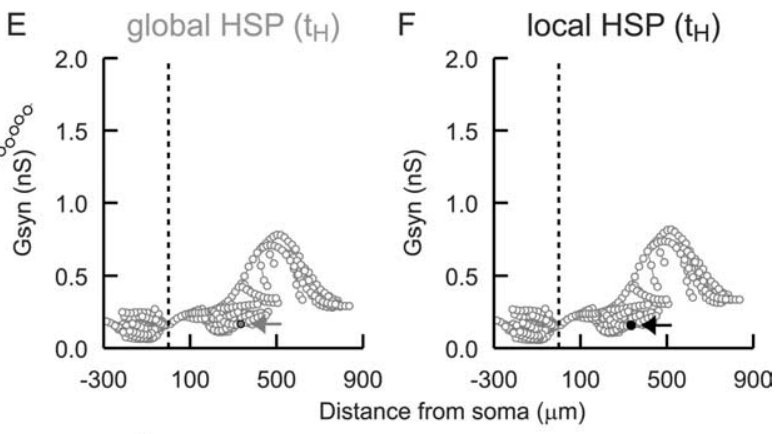

G

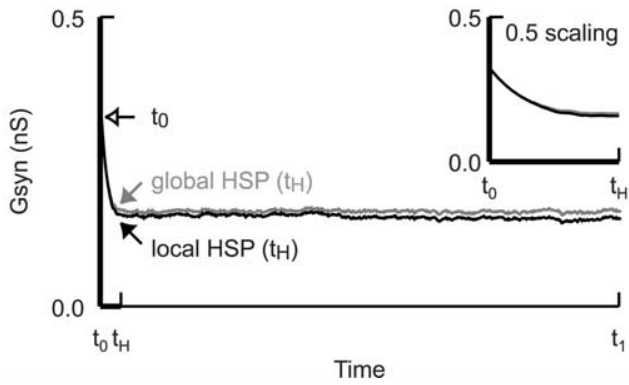

Figure 2. The time course of HSP after a massive overall increase in activity in a detailed model of a CA1 pyramidal neuron. $\boldsymbol{A}$, Average membrane potential, $\left\langle V_{i}\right\rangle$, at each synaptic site, $i$ (circles) for a uniform random activation of all synapses (circles in $\boldsymbol{D}$ ) at an average rate of $2 \mathrm{~Hz}$ before HSP (top profile) and after $/$-HSP (bottom profile). Horizontal dashed line marks the target membrane potential set at $V_{\text {trg }}=-60 \mathrm{mV}$. B, Peak synaptic conductance, $G_{\text {syn }, i}$, for each synapse, $i$ (circles) before HSP. All synapses had initially the same $G_{\text {syn }, i}=1 \mathrm{nS}$. C, $G_{\text {syn }, i}$ distribution after $/$-HSP. The $G_{\text {syn }, i}$ of a representative synapse (dark circle) is indicated by an open arrow. The time at which this $G_{\text {syn, } i}$ distribution was obtained is marked as $t_{0}$ (see below). D, Reconstructed CA1 pyramidal neuron (kindly provided to us by N. Spruston). Circles mark the locations of 587 equally spaced (20 $\mu \mathrm{m}$ intervals) excitatory synapses on the dendrites. Schematic pipette indicates the representative synapse from $\boldsymbol{C} . \boldsymbol{E}, \boldsymbol{F}, \boldsymbol{G}_{\mathrm{syn}, i}$ distributions afte $\mathrm{HSP}$ in response to an overall increase in activity produced by doubling the average synaptic activation rate from 2 to $4 \mathrm{~Hz}$ starting from time $t_{0}$ for $g$-HSP $(\boldsymbol{E})$ and for $l$-HSP $(\boldsymbol{F})$. The $G_{\text {syn }, i}$ of the representative synapse is indicated in each plot by an arrow. In both ses, HSP scaled down $G_{\text {syn, } i}$ values by half by the time marked as $t_{\mathrm{H}} . \mathbf{G}$, Time course of HSP downscaling of the $G_{\text {syn,i }, \text { of }}$ arrows point at the $G_{\text {syn }, i}$ value at time $t_{H}$ (corresponding to $\boldsymbol{E}$ and $\boldsymbol{F}$ ). The traces are shown for an additional prolonged period of time, $t_{1}$. The inset presents the two traces for the time between $t_{0}$ and $t_{\mathrm{H}}$, when most of the HSP scaling of $G_{\text {syn, } i}$ took place.

tions, we used a reconstructed model of a CA1 pyramidal neuron (Fig. 2D) with active channels in the axon and dendrites and multiple (587) excitatory synapses spaced at equal intervals of 20 $\mu \mathrm{m}$ throughout the dendrites (Fig. $2 D$, circles) (see Materials and Methods). The peak synaptic conductance, $G_{\text {syn, } i}$, of each synapse, $i$, was continuously modified according to the HSP mechanism described in Equation 1. The two possible variants of HSP were formulated. The first, $g$-HSP equally changed all synaptic weights according to the difference between the soma membrane potential, $V_{\text {soma }}$ (a possible global measure of activity) and a target membrane potential, $V_{\text {trg }}$ :

$$
\tau_{\mathrm{H}} \cdot \frac{d G_{\mathrm{syn}, i}}{d t}=\frac{V_{\mathrm{trg}}-V_{\mathrm{soma}}}{\kappa} \cdot G_{\mathrm{syn}, i},
$$



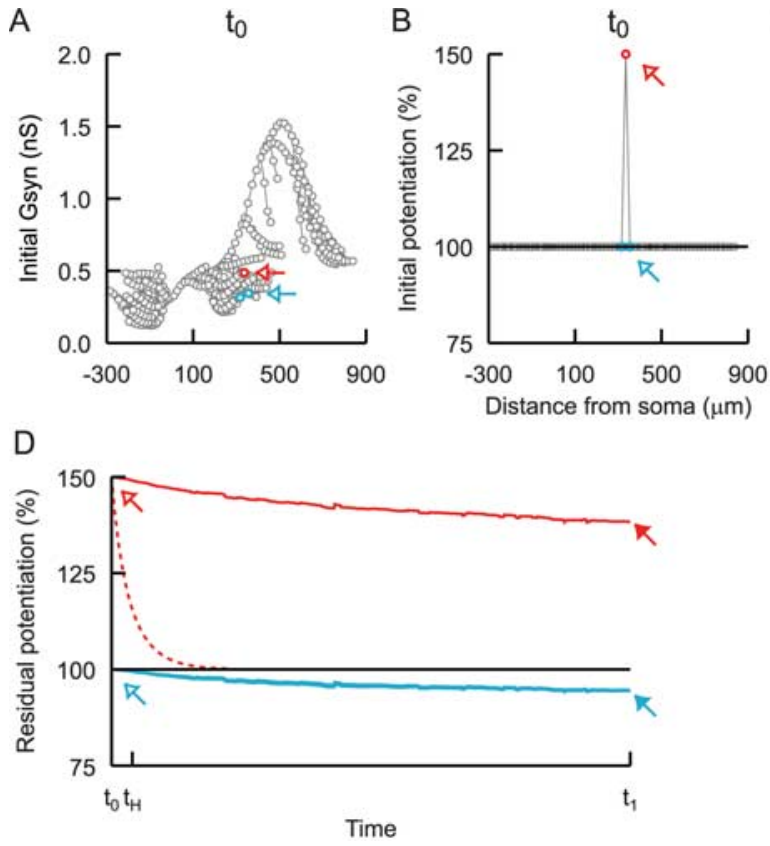

Figure 3. Residual potentiation of a single potentiated dendritic synapse after HSP. $A$, The initial $G_{\text {syn, },}$ distribution at time $t_{0}$ similar to Figure $2 C$, except for a $150 \%$ potentiation of $G_{\text {syn, } i}$ of the representative synapse (red circle and open arrow). The two immediate neighbors of the potentiated synapse are marked in blue. $\boldsymbol{B}$, The initial potentiation at time $t_{0}$, obtained by dividing all the $G_{\text {syn, },}$ values in $A$ by the corresponding values before potentiation in Figure $2 C$. C, The residual potentiation after $I-H S P$ at time $t_{1}$, obtained by dividing all $G_{\text {syn, },}$ values at time $t_{1}$ in the potentiated condition by the corresponding values in a control condition. D, Time course of the residual potentiation of the representative potentiated synapse (red) and of the suppression of its two immediate neighboring synapses (blue). Also plotted, for comparison, is the decay in the initial potentiation of a single synapse in the hypothetical case of an isolated compartment (dashed red trace, as in Fig. $1 B$ ). $\boldsymbol{E}$, The residual potentiation after postsynaptic I-HSP at time $t_{1}$, for a presynaptic $150 \%$ potentiation of the representative synapse (average activation rate of that synapse was raised from 2 to $3 \mathrm{~Hz}$ ).

Note that the conductance of all synapses changes according to the exact same expression on the right side of Equation 2. One could postulate a biological mechanism that can implement this, for example by changing the rate of relevant mRNA transcription at the cell nucleus with respect to somatic levels of activity.

The second, $l$-HSP controlled the local membrane potential, $V_{i}$, at each synaptic site, $i$ ( $V_{\text {trg }}$ was assumed to be identical for all dendritic sites):

$$
\tau_{\mathrm{H}} \cdot \frac{d G_{\mathrm{syn}, i}}{d t}=\frac{V_{\mathrm{trg}}-V_{i}}{\kappa} \cdot G_{\mathrm{syn}, i},
$$

In this scheme, each synapse changes differentially according to the local level of activity, which reflects how activity transpiring throughout the dendritic branch is integrated at the site of that synapse. Biologically, this could occur through local control of protein synthesis at the site of each synapse (Sutton et al., 2004, 2006).

With all synapses set initially at a uniform peak conductance of $G_{\text {syn }, i}=1 \mathrm{nS}$ (Fig. $2 \mathrm{~B}$ ) and each synapse activated randomly and asynchronously at $2 \mathrm{~Hz}$, the average membrane potential at each synaptic site, $\left\langle V_{i}\right\rangle$, assumed a very non-uniform shape (Fig. $2 A$, "prior to HSP"). We set $V_{\text {trg }}=-60 \mathrm{mV}$ (Fig. $2 A$, horizontal dotted line) and applied the $l$-HSP rule to all dendritic synapses. After $l$-HSP, $\left\langle V_{i}\right\rangle$ values all leveled at the $V_{\text {trg }}$ line (Fig. $2 \mathrm{~A}$, "local HSP") and the emergent distribution of synaptic weights became highly non-uniform (Fig. 2C), with a distance-dependent increase in synaptic strength along the initial $500 \mu \mathrm{m}$ of the main apical trunk, reminiscent of that found in CA1 pyramidal neurons (Magee and Cook, 2000). We demonstrated previously
(Rabinowitch and Segev, 2006) that $g$-HSP, in contrast to $l$-HSP, will proportionally scale up or down the initial distribution of synaptic weights and will not necessarily level all $\left\langle V_{i}\right\rangle$ values to the desired $V_{\text {trg }}$ level. This also occurred in the example presented in Figure 2 (data not shown).

To appreciate the model HSP timescale, we ran a simulation similar to the typical experimental paradigm used to study HSP, in which the activity of a neuron is globally enhanced, e.g., by pharmacologically reducing inhibitory synaptic input to the neuron (Lissin et al., 1998; O’Brien et al., 1998; Turrigiano et al., 1998). In the model, this was implemented by doubling the average frequency of synaptic activation from 2 to $4 \mathrm{~Hz}$, starting with the initial spatial distribution of synaptic weights as in Figure $2 C$, in which $\left\langle V_{i}\right\rangle=V_{\text {trg }} \cdot g$-HSP and $l$-HSP responded to such overall perturbation similarly (Rabinowitch and Segev, 2006), by uniformly scaling down all synaptic weights (Fig. $2 E, F$ ). The time course of the change in conductance of a representative synapse (indicated in Fig. $2 D$ by the schematic pipette and by arrows in Fig. $2 C, E, F)$ is plotted in Figure $2 G$. As can be seen, most of the downscaling was completed for both $l$-HSP and $g$-HSP by time $t=t_{\mathrm{H}}$ (Fig. $2 G$, inset). We continued the simulation for a long time beyond $t_{\mathrm{H}}$, until $t=t_{1}$. During this time, there was not much additional change in $G_{\text {syn }, i}$. Thus, under conditions of overall perturbation of activity, the time between $t_{0}$ and $t_{\mathrm{H}}$ can be considered to be equivalent to the many hours to days time course of HSP under comparable experimental conditions.

\section{Residual potentiation of a single potentiated dendritic synapse}

Research on LTP has contributed an immensely rich diversity of both theoretical and experimentally founded rules for the induction of LTP (Sjostrom et al., 2001; Abarbanel et al., 2003; Froemke et al., 2005). However, these rules vary greatly, and, in addition, the dependence of LTP induction on dendritic location is only beginning to be understood (Froemke et al., 2005). We therefore decided not to implement a specific activity-dependent LTP rule in our model but instead to assume that certain synapses underwent potentiation and to artificially increase the strength (peak conductance) of these potentiated synapses by some initial value, $p^{0}$. This has the additional benefit of rendering our conclusions independent of the exact mechanism governing LTP induction.

Figure 3, $A$ and $B$, shows an initial potentiation of the representative synapse from Figure $2 C$ to $p^{0}=150 \%$ at time $t=t_{0}$ (Fig. $3 A, B$, potentiated synapse in red and immediate two neighboring synapses in blue). As in Figure $2 C$ (and unless otherwise mentioned), all synapses were activated randomly at $2 \mathrm{~Hz}$. The potentiation of this single synapse (of the large number of synapses occupying the dendrites), located $330 \mu \mathrm{m}$ from the soma, went unnoticed by $g$-HSP (data not shown) and will thus persist 
indefinitely. In contrast, $l$-HSP responded by weakening the initial potentiation of the synapse to a residual potentiation of $\sim 140 \%$ (Fig. 3C, red). In addition, it also slightly weakened the nearby nonpotentiated synapses (Fig. 3C, blue). This "lateral suppression" created a new balance, allowing the potentiated synapse to maintain its potentiation and thus supporting it. The time course for the drop in residual potentiation is plotted in Figure $3 D$ (solid red trace). Also plotted, for comparison, is the decay in residual potentiation in the isolated case (dashed red trace, reproduced from Fig. $1 B$ ) and the suppression of the two neighboring synapses (blue overlapping traces). Recall that $t=t_{1}$ is equivalent to a very long time period in real time (compare with Fig. 2G). Thus, we find that the potentiation of a single synapse can persist even in the presence of an $l$-HSP mechanism, thanks to the slight weakening of its nearby nonpotentiated neighboring synapses. How does such supporting lateral suppression come about? This predicted phenomenon is attributable to the distance-dependent interactions occurring along the dendritic branch. The potentiation of a synapse $i$ (Fig. 3B, red synapse) will naturally result in an increase in $\left\langle V_{i}\right\rangle$ at the site of that synapse but also in an increase in $\left\langle V_{i}\right\rangle$ in neighboring sites $j$ (Fig. $3 B$, blue synapses), causing $l$-HSP to weaken these neighboring synapses together with synapse $i$. The weakening of the neighboring synapses will, in turn, contribute to driving $\left\langle V_{i}\right\rangle$ values back to $V_{\text {trg }}$, forming a new stable constellation of synaptic weights as in Figure 3C.

The expression of LTP could also occur presynaptically (Reid et al., 2004). In Figure 3E, we demonstrate that the same principle shown in Figure $3 C$ also holds for this case. We "potentiated" the representative synapse presynaptically by increasing its average activation rate by $150 \%$ (from 2 to $3 \mathrm{~Hz}$ ). This could be interpreted, for example, as an increase in release probability. In principle, the complete abolishment of this potentiation by a postsynaptic HSP mechanism would be the reduction of the peak synaptic conductance to $1 / 1.5=66.7 \%$ of its original strength. $l$-HSP indeed weakened the conductance of the potentiated synapse, but only to $90 \%$ (Fig. $3 E$, red). Once again, lateral suppression hindered any additional loss of the potentiation of the synapse.

\section{Residual potentiation of a group of potentiated dendritic synapses}

What happens in an electrically distributed dendrite when more than just one synapse is potentiated? As we have seen in the isopotential case, the number of potentiated synapses, $n_{p}$, compared with the total number of synapses, $N$, is of key importance in determining the residual potentiation. Is this also true for the realistic neuron?

In Figure 4, we present the residual potentiation after $l$-HSP of the representative synapse from Figure 3 under three different conditions: (1) the reference case in which only that synapse had been potentiated (Fig. $4 A$, left, $B$, red trace as in Fig. $3 D$ ); (2) a
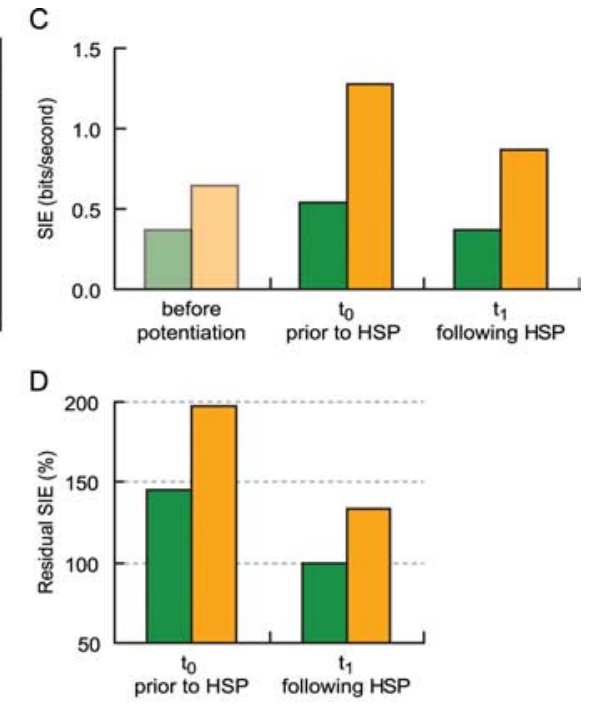

D

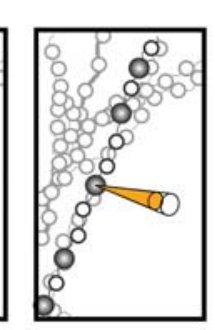

Figure 4. Residual potentiation after /-HSP depends on both the number of potentiated synapses and their spatial distribution.

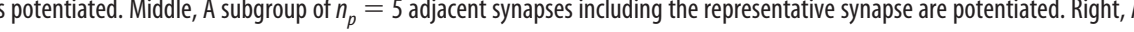
their steady-state values as in Figure $2 C$; after a $p^{0}=150 \%$ potentiation of the subgroup synapses at time $t_{0}$ before HSP (" $\mathrm{t}_{0}$ the SIE values for each condition by the corresponding SIE values before potentiation.

total of $n_{p}=5$ adjacent synapses, including the representative synapse (Fig. $4 A$, middle), were all potentiated to $p^{0}=150 \%$; this increase in $n_{p}$ resulted in a big reduction in the residual potentiation of the synapse (Fig. $4 B$, green trace); and (3) the same number $\left(n_{p}=5\right)$ of synapses was potentiated, but this time the synapses that underwent potentiation were more spread out (Fig. $4 A$, right); now the residual potentiation was larger (Fig. $4 B$, compare orange trace with green trace).

Although consisting of the same number of synapses $\left(n_{p}=5\right)$, the "clustered" (Fig. 4A, middle) and "dispersed" (Fig. 4A, right) patterns are distinguishable at the level of the dendritic branch and were thus subject to different levels of suppression by $l$-HSP (Fig. $4 B$, green and orange traces). Are these local variations in spatial distribution also detectable by the soma? What is their impact on the output of the postsynaptic neuron?

To address these questions, we designed a simulation in which the input from the subgroup of the five potentiated synapses transiently changes to become synchronized and its activation rate is increased from 2 to $10 \mathrm{~Hz}$ (the rest of the synapses remain at $2 \mathrm{~Hz}$ ). This momentary change could represent some sort of event that the postsynaptic neuron is expected to detect, occurring at the short timescale (too brief for HSP to respond to). To assess the impact of the representative synapse on the firing of the neuron within different spatial patterns, we used the SIE measure (London et al., 2002), which estimates the mutual information (in bits per second) between the presynaptic and postsynaptic firing patterns (see Materials and Methods). First, we compared between the SIEs of the clustered (green) and dispersed (orange) patterns before any synaptic potentiation (Fig. 4C, "before potentiation"). In this case, the representative synapse and its four adjacent neighbors (Fig. $4 A$, middle) or four scattered neighbors (Fig. $4 A$, right) received the enhanced synchronized input with 


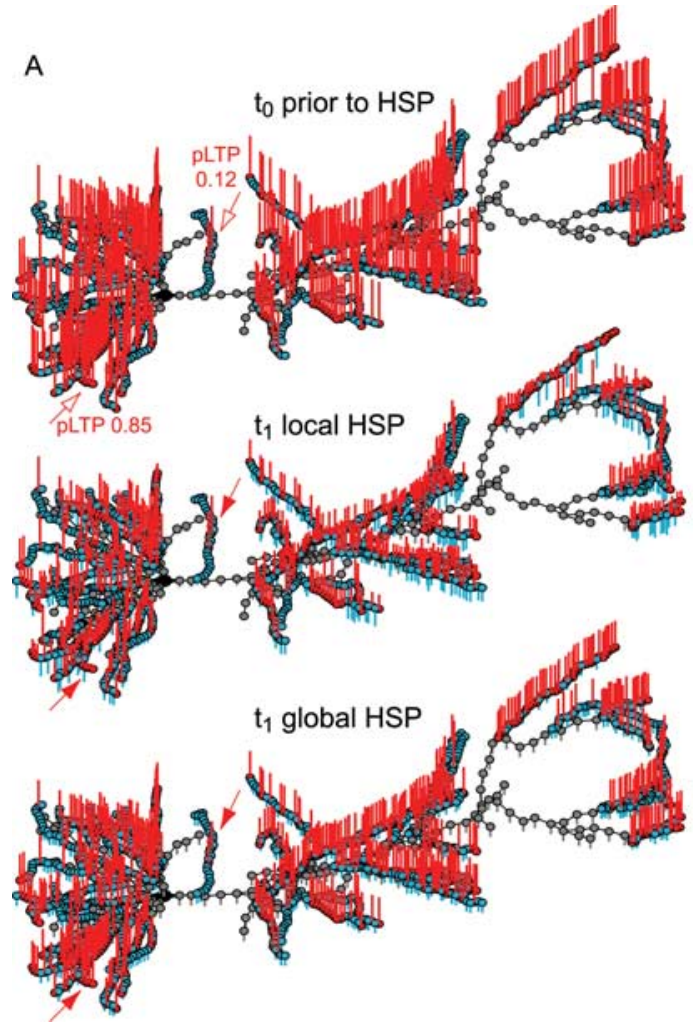

B


to prior to HSP

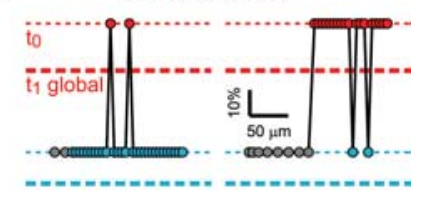

$t_{1}$ local HSP

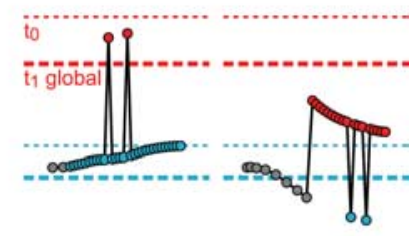

$t_{1}$ global HSP



Figure 5. HSP selecting between different spatial patterns of synaptic potentiation. $\boldsymbol{A}$, Top, Synapses occupying the 50 longest dendritic terminals were randomly selected for potentiation according to a branch-specific probability for potentiation, $p \mathrm{LTP}$. In these branches, synapses were densely spaced $5 \mu \mathrm{m}$ from one another. The potentiated synapses are shown in red ( $p^{0}=150 \%$ for all potentiated synapses, represented by the red bars). The neighboring nonpotentiated synapses are in blue. The rest of the synapses on the tree (spaced at $25 \mu \mathrm{m}$ intervals) are in gray. Open arrows point at two representative branches, an oblique branch with $p L T P=0.12$ and a basal branch with $p L T P=0.85$. The amount of residual potentiation is represented by the size of the red bars, and the extent of suppression of the nonpotentiated synapses is represented by the size of the blue and gray downward pointing bars, after /-HSP (middle) and $g$-HSP (bottom). B, Residual potentiation levels of the two representative branches (from $A$; left, oblique branch with $p L T P=0.12$; right, basal branch with $p L T P=0.85$ ) for time $t_{0}$ (top, potentiated synapses aligned with top dotted red lines, labeled " $\mathrm{t}_{0}{ }^{\prime}$ ); for time $t_{1}$ after $/$-HSP (middle); and after $g$-HSP (bottom, potentiated synapses aligned with the $g$-HSP dashed thick red lines, labeled " $\mathrm{t}_{1}$ global"). Nonpotentiated synapses are also shown (blue and gray circles as in $\boldsymbol{A}$ ). Middle dotted blue lines mark $100 \%$ of original synaptic strength, and bottom dashed thick blue lines mark the level of suppression after $g$-HSP.

all synaptic weights as in Figure 2C. Even before any potentiation was introduced, the two spatial patterns were discernable at the soma as measured by the corresponding SIEs. The representative synapse was 1.75 more effective in influencing the firing of the postsynaptic neuron when participating in the dispersed subgroup than in the clustered one (compare between the light orange and green columns in Fig. $4 C$, "before potentiation"). The smaller synaptic efficacy in the clustered case can be explained by the reduction in the driving force and local input resistance of the synapses attributable to the spatial proximity of the synapses.

The $p^{0}=150 \%$ potentiation of the synapses in the subgroup substantially enhanced the SIE in both the clustered (green) and dispersed (orange) conditions (Fig. $4 C$, " $t_{0}$ prior to HSP"). The $150 \%$ potentiation of the five synapses resulted in an $\sim 150 \%$ increase in the SIE in the clustered case (compare leftmost and middle green columns in Fig. $4 C$ whose ratio is shown in Fig. $4 D$, green column, " $t_{0}$ before HSP") and an $\sim 200 \%$ increase for the dispersed case (Fig. $4 D$, orange column, " $\mathrm{t}_{0}$ before HSP"). After l-HSP, which slowly weakened the weights of the potentiated synapses (Fig. $4 B$ ), the SIE also decreased for both patterns (Fig. $4 C$, " $t_{1}$ following HSP"). However, in the clustered case, the SIE dropped to its value before potentiation (Fig. $4 D$, green column,
" $t_{1}$ following HSP"), whereas in the dispersed pattern, there remained a substantial amount of residual SIE (133\%) after HSP (Fig. 4D, orange column, " $t_{1}$ following HSP").

Thus, in the realistic neuron, under l-HSP, it is not only the number of potentiated synapses, $n_{p}$, that matters but also their spatial distribution. At the large timescale, $l$-HSP will favor synaptic potentiations that occur within a sparse spatial pattern of potentiations over a dense pattern. Coincidently, at the short timescale, a synapse pertaining to such a sparse pattern, when activated in synchrony with its partners, will have a stronger impact on the firing of the neuron. In contrast to $l$-HSP, $g$-HSP did not have the spatial resolution to distinguish between the two patterns in the above example (data not shown). An extensive comparison between $l$-HSP and $g$-HSP follows.

\section{The survival of massive synaptic} potentiations under HSP in dendrites We further explored the effect of both $l$-HSP and $g$-HSP on varied spatial patterns of synaptic potentiations. In our model neuron, we selected the 50 longest terminal branches in the dendritic tree and substantially increased the number of synapses on these branches by decreasing the interval between them to $5 \mu \mathrm{m}$ (increasing the total number of excitatory synapses on the dendrites to 1514) (see Materials and Methods). We ran a $l$-HSP simulation under these conditions to obtain the initial distribution of synaptic conductances (data not shown). For each branch, a probability for potentiation, pLTP, was drawn from a uniform distribution ranging from 0.1 to 0.9 , and each synapse along the branch was potentiated with that probability to $150 \%$ of its initial strength. All in all, 591 of a total of 1514 synapses were potentiated $\left(n_{p} / N>1 / 3\right)$. This is illustrated in Figure $5 A$ (top), in which the red filled circles represent the synapses that were potentiated (the red bars rising from them represent $150 \%$ potentiation). The blue filled circles represent the nonpotentiated synapses sharing the same branches, and the gray filled circles represent the rest of the synapses on other parts of the dendritic tree. Two representative branches are indicated by the open arrows: an oblique branch with $p \mathrm{LTP}=0.12$ had just two potentiated synapses (of 30) and a basal branch with pLTP $=0.85$ had 18 potentiated synapses (of 20). The residual potentiation of these synapses after a long time, $t=t_{1}$, is presented for both $l$-HSP (Fig. $5 A$, middle) and $g$-HSP (Fig. $5 A$, bottom). We ran these simulations for an additional equally long period of time (until $t=t_{2}$ ) and compared between the residual potentiations at $t_{2}$ and $t_{1}$. They showed a clear match for both $g$-HSP and l-HSP (data not shown), indicating that these were steady-state residual potentiations.

The length of the red bars in Figure $5 A$ is proportional to the residual potentiation, decreased in both cases attributable to the 
overall large number of potentiated synapses $\left(n_{p} / N>1 / 3\right)$. In addition, under both HSP variants, many neighboring synapses on the selected branches and throughout other parts of the dendrites also weakened (downward pointing blue and gray bars). However, although the level of residual potentiation was uniform for all synapses under $g$-HSP (132\%) (Fig. $5 B$, bottom marked by thick red dashed lines), it was strongly branch specific under $l$-HSP. This can be appreciated for the two representative branches. The oblique branch with $p \mathrm{LTP}=0.12$ was only mildly affected by $l$-HSP (Fig. $5 B$, middle left). In contrast, the synapses on the basal branch with $p \mathrm{LTP}=0.85$, lost most of their initial potentiation (Fig. 5B, middle right). Thus, l-HSP, unlike g-HSP, will differentially and selectively affect the residual potentiations of synapses. Because l-HSP can operate at the level of functional dendritic compartments (Rabinowitch and Segev, 2006), it will be sensitive to the relative number of potentiated synapses $\left(n_{p, k} / N_{k}\right)$ within each given compartment, $k$, whereas the $g$-HSP mechanism will respond to the total number of potentiated synapses in the entire tree $\left(n_{p} / N\right)$.

\section{Predicting the residual potentiation from the initial spatial pattern of synaptic potentiations}

Can information on the remaining residual potentiation after HSP be extracted from the initial spatial pattern of synaptic potentiations? Figure $6 \mathrm{~A}$ presents the residual potentiations of the synapses from Figure 5 as a function of their initial level of potentiation. Although all potentiated synapses shared the same level of initial potentiation, $p^{0}=150 \%$, they exhibited a very wide range of residual potentiations under l-HSP (Fig. 6A, vertical red bar showing residual potentiation values ranging approximately between 100 and $150 \%$ ) and a particular uniform level of residual potentiation of $132 \%$ under $g$-HSP (Fig. $6 A$, thick dashed red line). Similarly, the nonpotentiated synapses in Figure 5 began at $100 \%$ of their strength at time $t_{0}$ and were suppressed by $l$-HSP to levels ranging approximately between 70 and $100 \%$ of their initial strength (Fig. $6 A$, blue vertical bar) and to $88 \%$ of their initial strength by $g$-HSP (Fig. $6 A$, thick dashed blue line). Thus, the residual potentiation cannot be predicted from the initial potentiation levels alone. What additional information is needed?

Although under $g$-HSP all synaptic weights are modified in unison, this does not necessarily mean that all patterns of synaptic potentiations will be scaled by the same factor (as in the case of the isopotential neuron). In effect, in an electrically distributed neuron, the influence of synaptic potentiation on the global activity level [such as the value of $V_{\text {soma }}$ or the average rate of action potential (AP) firing] is expected to vary according to the spatial distribution of these potentiations. For example, $n_{p}$ potentiated
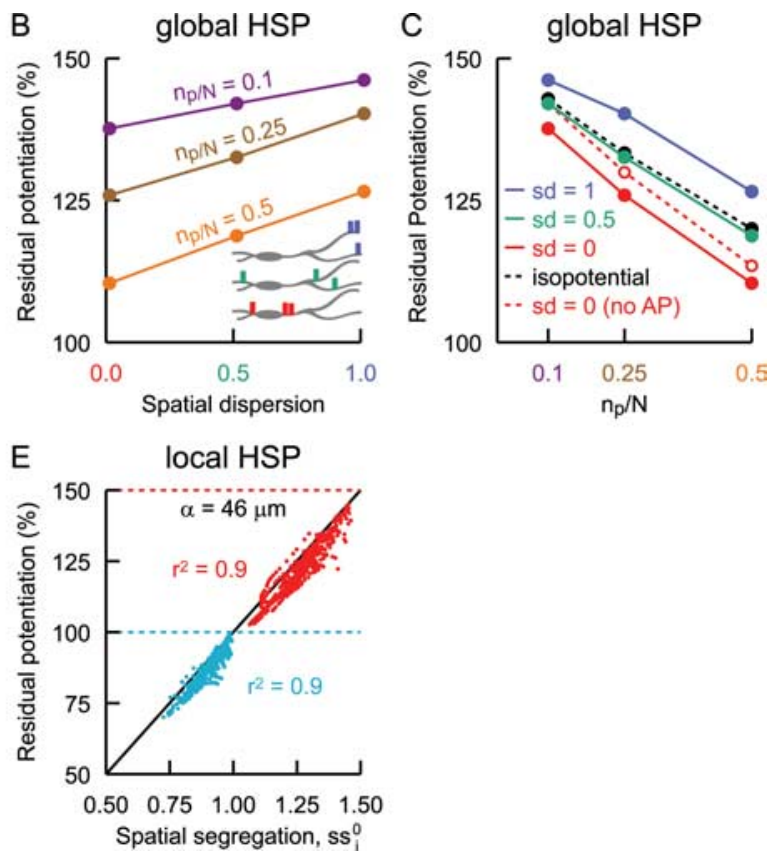

Figure 6. Predicting the residual potentiation from the initial spatial pattern of synaptic potentiations. $\boldsymbol{A}$, Residual potentiation $g$-HSP (thick blue dashed line). The diagonal is the equality line. $\boldsymbol{B}$, Residual potentiation at time $t_{1}$ (with initial synaptic conduc列 $N=0.1,0.25$, and 0.5 ). Inset, Schematic examples of the three different spatial dispersion configurations used (bars represen p potentiated synapses, $n_{p} / N$. Each curve represents a different spatial pattern of synaptic potentiations with varying spatial disper(SD $=1,0.5$, and 0 , in cyan, turquoise, and red, respectively). Also plotted is the theoretical curve for the isopotential case which action potentials were blocked (dashed red, "no AP"). D, Average residual potentiation (from Fig. 5) of the synapses at each synapse at timet after /HSP plottedagainst the spatialsegregationindex, ss (supplementa Appendix II, Equation A12, at www.jneurosci.org as supplemental material), for that synapse with $\alpha=46 \mu \mathrm{m}$ (see Results; each dot represents a synapse). Red and blue represent the potentiated and nonpotentiated synapses, respectively. Diagonal is the equality line.

synapses that are clustered close to the soma will have a much greater influence on $V_{\text {soma }}$ than if the same number of potentiated synapses are located distally, at remote dendritic arbors. Therefore, even under a $g$-HSP mechanism (that is sensitive to the level of activity at the soma), the spatial pattern of the potentiated synapses is expected to determine the degree of their remaining residual potentiations. In Figure 6, $B$ and $C$, we present quantitatively the influence of the extent of the spatial dispersion of a given pattern of synaptic potentiations on the remaining residual potentiation after $g$-HSP, using a measure for the degree of spatial dispersion, $\mathrm{SD}_{j}$, for a specific spatial pattern of potentiations, $j$ (supplemental Appendix II, Equation A11, available at www.jneurosci.org as supplemental material). $\mathrm{SD}_{j}$ may vary between 0 , indicating no dispersion (i.e., all potentiated synapses are located as close as possible to the soma) (Fig. 6B, inset, bottom configuration), and 1, indicating maximal dispersion (i.e., all potentiated synapses are as distant as possible from the soma) (Fig. $6 B$, inset, top configuration). Intermediate values correspond 
to intermediate configurations (Fig. 6B, inset, middle configuration).

We ran several simulations using the model from Figure 2, with initial $G_{\mathrm{syn}, i}$ as in Figure $2 B$, for various spatial potentiation patterns with different spatial dispersion indices $\left(\mathrm{SD}_{j}=0,0.5,1\right)$ and with different numbers of synapses initially potentiated to $p^{0}=150 \%\left(n_{p} / N=0.1,0.25\right.$, and 0.5$)$. Figure $6, B$ and $C$, depicts the residual potentiations after $g$-HSP for these simulations. The residual potentiation under $g$-HSP was indeed larger with increased spatial dispersion (Fig. $6 \mathrm{~B}$ ). At the same time, an increase in $n_{p} / N$ reduced the size of the residual potentiation. Thus, both the number of potentiated synapses and their spatial distribution throughout the entire dendritic tree will determine the size of the residual potentiation after $g$-HSP. In Figure $6 C$, we plotted the residual potentiation as a function of the number of potentiated synapses for each spatial dispersion pattern and compared these plots with the theoretical curve for the isopotential case (dotted black line; reproduced from Fig. $1 C$, for the $p^{0}=150 \%$ case). The extremely peripheral potentiation patterns $\left(\mathrm{SD}_{j}=1\right.$; cyan filled circles) showed larger residual potentiations than the corresponding potentiations of the same number of synapses in an isopotential neuron (black filled circles). The extremely somatocentric potentiation patterns $\left(\mathrm{SD}_{j}=0\right.$, red filled circles) had smaller residual potentiations than in the corresponding isopotential case. The intermediate potentiation patterns $\left(\mathrm{SD}_{j}=0.5\right.$, turquoise filled circles) resulted in residual potentiations that were very similar to isopotential levels. We ran an additional set of simulations for the somato-centric distribution $\left(\mathrm{SD}_{j}=0\right)$ in which AP generation was blocked (Fig. $6 C$, red open circles and dotted line). This revealed the significant contribution that the increased AP firing rate (attributable to the potentiation of proximal synapses) had to the weakening of the residual potentiations in the control simulation (Fig. $6 C$, solid red line). However, even in the absence of APs, the residual potentiation of the somatocentric potentiation patterns was still distinguishable from that of the peripheral patterns (compare red open circles with cyan filled circles). The lack of APs had no significant impact on the residual potentiations for the intermediate $\left(\mathrm{SD}_{j}=0.5\right)$ and peripheral $\left(\mathrm{SD}_{j}=1\right)$ configurations (data not shown).

We conclude that a $g$-HSP mechanism, based on activity at the soma, will weaken synaptic potentiations according to their spatial distribution throughout the dendrites. $n_{p}$ potentiated synapses spatially distributed in a somato-centric pattern will be weakened more than an equal number of potentiated synapses in the corresponding isopotential neuron. Indeed, in an electrically distributed dendritic tree, the influence of proximal potentiated synapses, relative to the other nonpoteniated ones, is greater than in the corresponding isopotential case. For the same reasons, a peripheral pattern of potentiated synapses will be weakened less than in an isopotential neuron.

Under l-HSP, as we have seen (Figs. 4, 5A, middle, 5B, middle), the amount of weakening of potentiated synapses is mainly determined at the level of the dendritic branch, according to the degree of sparseness of the potentiated synapses on that branch. As a first approximation for this branch-specific sparseness, we chose the probability of potentiation, $\mathrm{LTP}_{k}$, of synapses on branch $k$. This measure is related to the ratio of the number of potentiated synapses to the total number of synapses, $n_{p, k} / N_{k}$, used to analytically predict the residual potentiation in the isopotential case (Fig. $1 C$ ). In dendritic trees, the $p \mathrm{LTP}_{k}$ we applied also reflects the spatial distribution of the potentiated synapses (e.g., with low $p \mathrm{LTP}_{k}$, the chances that the potentiated synapses will be spatially segregated is high). In Figure $6 D$, we plotted the average residual potentiation, $\langle\mathrm{rp}\rangle$, of the potentiated synapses from Figure $5 A$ (middle) on each branch against the $p$ LTP of that branch (red dots). The theoretical curve from Figure $1 C$, for an initial potentiation of $p^{0}=150 \%$, is also plotted (red line). The match was remarkable $(>70 \%$ of the variance of $\langle\mathrm{rp}\rangle$ could be explained by the theoretical curve). We repeated this for an initial potentiation of $p^{0}=200 \%$ (green dots and line) and an initial depression of $50 \%$ (orange dots and line) and obtained similarly good matches with the theoretical curves. Thus, in each dendritic branch undergoing $l$-HSP, the degree of retention of $n_{p}$ synaptic potentiations that are well spaced is equivalent to that of any $n_{p}$ synaptic potentiations in an isopotential dendrite. However, as we have shown (Fig. 4), if the same number of synaptic potentiations are clustered, then the residual potentiation will be much smaller than in the isopotential case.

Finally, we sought a measure that could predict the residual potentiation of each individual synapse after l-HSP in the dendritic tree. In electrically distributed dendrites, not only $n_{p}$ matters but also the distance of the $n_{p}$ potentiated synapses one from another. Therefore, we defined an estimator, $s s_{i}^{0}$ (supplemental Appendix II, Equation A12, available at www.jneurosci.org as supplemental material), for the residual potentiation of each potentiated synapse $i$, based on the spatial segregation of the initial potentiation of that synapse. This spatial segregation index, $s s_{i}^{0}$, is an average of the ratio

$$
\frac{p_{i}^{0}}{p_{j}^{0}}
$$

of the initial potentiation of synapse $i$ and that of each one of its neighbors, $j$, weighted according to the distance between these synapses. Importantly, the definition of $s s_{i}^{0}$ includes a free parameter, $\alpha$ (in micrometers), reflecting the size of the region that influences the fate of each potentiated synapse within the dendritic branch.

$s s_{i}^{0}$ will equal 1 (predicting a complete loss of potentiation, $\mathrm{rp}=100 \%)$ in either the case in which all synapses on the branch are equally potentiated $\left(p_{i}^{0}=p^{0} \forall i\right)$ or the case in which there are only very few synapses on that branch. In contrast, $s s_{i}^{0}$ will equal $p_{i}^{0}$ (predicting full conservation of the initial potentiation, $\mathrm{rp}=$ $p_{i}^{0}$ ) if synapse $i$ is the only potentiated synapse on a crowded branch (the rest of the synapses have $p_{i}^{0}=100 \%$ ).

In Figure $6 E$, the actual residual potentiation of each potentiated synapse (from Fig. $5 \mathrm{~A}$, middle) is plotted against the spatial segregation estimator, $s s_{i}^{0}$ for $\alpha=46 \mu \mathrm{m}$ (red dots). The correlation was excellent $\left(r^{2}=0.9\right)$. We also plotted the suppression of the nonpotentiated synapses against their $s s_{i}^{0}$ and obtained an equally good correlation (blue dots). The value of $\alpha$ (supplemental Appendix II, Equation A12, available at www.jneurosci.org as supplemental material) was chosen so as to maximize the correlation coefficient, $r^{2}$. This "effective region of influence," in the order of several tens of micrometers, seems to be typical for the interaction between synapses in dendritic branches (Engert and Bonhoeffer, 1997). Increasing the interval between the synapses (and thus decreasing their number) did not affect the optimal $\alpha$ because, as we mentioned, $\alpha$ reflects the size of the relevant dendritic region influencing synapse $i$ and not the number of synapses occupying this region. In contrast, increasing the effective electrical space constant of the dendrites, $\lambda$ (e.g., by reducing the axial resistance, $R_{i}$, or by "blocking" the voltage-sensitive ion channels in this branch) increased the optimal value for $\alpha$ (data not shown). In conclusion, under l-HSP, a simple distanceweighted average of the initial synaptic potentiations along the 
dendritic branch is sufficient for predicting the residual potentiation of each synapse.

\section{Discussion}

To date, the most widely studied form of synaptic plasticity is undoubtedly LTP/LTD. However, the actual spatial distribution of synaptic weights on the dendrites of a neuron is shaped by the joint actions of several different forms of synaptic plasticity working in parallel with LTP/LTD. Several studies have alluded to this issue (Bienenstock et al., 1982; Miller, 1996; Abbott and Nelson, 2000; Goldberg et al., 2002) and specifically to the cooperation between LTP and homeostatic synaptic plasticity (Turrigiano and Nelson, 2000; van Rossum et al., 2000; Kempter et al., 2001; Burrone and Murthy, 2003; Yeung et al., 2004; Toyoizumi et al., 2005), yet these works typically regard the neuron as a homogeneous unit lacking any spatial dimensions (but see Siegel et al., 1994). The main conclusion has been that a division of labor exists between short timescale activity-dependent "Hebbian" LTP/LTD processes and HSP, with the former in charge of molding the spatial pattern of synaptic potentiations, and the latter responsible for uniformly scaling the weights of these potentiated synapses up or down and thus stabilizing the activity of the neuron at the large timescale. In the present study, we sought to revisit this question, taking into consideration the important, so far mostly ignored, spatial dimension of the neuron and its implications on the spatial interactions between different synapses and between LTP/LTD and HSP.

It is unknown whether HSP is directly controlling postsynaptic membrane potential (Leslie et al., 2001; Paradis et al., 2001; Burrone et al., 2002; Moulder et al., 2003), calcium concentration (Thiagarajan et al., 2002; Yeung et al., 2004; Pawlak et al., 2005), or some other component of the activity of the neuron. We focused on membrane potential because its spread in dendrites has a well established theoretical framework (Rall, 1959) and because, in general, the average membrane potential $\left\langle V_{i}\right\rangle$ is a useful measure to capture the dynamics of neuronal activity (Koch et al., 1995). We argue that our conclusions also hold for a possible HSP mechanism based on calcium (or any other substance) that exhibits a distance-dependent diffusion along dendrites (notwithstanding its shorter space constant compared with voltage), thus providing a channel for the interaction between synapses situated at different dendritic locations (Zador and Koch, 1994; Eilers et al., 1995; Ross et al., 2005; Zelles et al., 2006). We note that, in this study, we assumed that $V_{\operatorname{trg}}$ (the target membrane potential) is a constant, arbitrarily chosen to be $60 \mathrm{mV}$. In principle, $V_{\text {trg }}$ could, in itself, be a modifiable parameter. This will very slowly change the operating set point of a neuron (increasing or decreasing its average firing rate). However, the results presented here (e.g., the persistent sparse patterns of LTP) hold also in face of this possibility.

In addition to HSP, other negative-feedback forms of synaptic plasticity have been suggested or shown to exist, such as synaptic depression during spike-timing-dependent plasticity (Song et al., 2000), metaplasticity (Bienenstock et al., 1982; Abraham and Bear, 1996), and anti-Hebbian forms of plasticity (Rumsey and Abbott, 2004). However, HSP can be distinguished from the others in its very slow dynamics (days) and in its independence on synaptic activation (Rao and Craig, 1997; O’Brien et al., 1998; Turrigiano et al., 1998; Leslie et al., 2001; Thiagarajan et al., 2005).

We found that the contribution of HSP to the shaping of the synaptic weight distribution is not limited to the normalization of already existing patterns of synaptic potentiations but rather that
HSP could be selecting between these patterns according to the nature of the mechanism underlying HSP. A global mechanism ( $g$-HSP), modifying all synaptic weights in unison according to a cell-wide measure of activity, such as the level of activity at the soma, will weaken synaptic potentiation patterns that are centered around the soma to a larger extent (because they will have a stronger influence on soma activity) than disperse potentiation patterns. This could serve perhaps as a device for "corrective discrimination" in the neuron, improving the impact of the distal, less influential, synaptic potentiations on the overall activity of the neuron, in addition to the distance-dependent scaling of synaptic strength along the main apical trunk of CA1 pyramidal neurons (Magee and Cook, 2000).

One could argue that the $g$-HSP mechanism is actually implemented by a local mechanism that is selectively sensitive to signals that are common to all synaptic sites, such as the backpropagating action potential (BPAP). However, action potentials are not necessary for HSP to occur (Leslie et al., 2001; Ju et al., 2004; Sutton et al., 2004, 2006), indicating that HSP is not selective to BPAPs, and, in addition, BPAPs are not uniform throughout the dendrites (Stuart et al., 1997; Goldberg et al., 2002; Lisman and Spruston, 2005). For instance, in CA1 pyramidal neurons, BPAPs attenuate as a result of a distance-dependent increase in A-type conductance (Johnston et al., 1999), they do not reach all branches of the dendrite (Golding et al., 2001), and they may well be confounded (Goldberg et al., 2002; Lisman and Spruston, 2005) with local dendritic spikes (Golding and Spruston, 1998; Ariav et al., 2003). Therefore, if HSP is indeed global, it is expected to have a qualitatively different underlying mechanism than $l$-HSP.

Indeed, if HSP is based on a mechanism that directly monitors the local level of activity at the site of each synapse (Sutton et al., 2006) and accordingly modifies the strength of that synapse, then it will show very different selectivity between potentiation patterns than that of $g$-HSP. We demonstrated previously that, under l-HSP, spatial patterns of synaptic potentiation can be preserved and scaled up or down by a branch-specific scaling factor (Rabinowitch and Segev, 2006). Here we have shown that the extent of this scaling will depend on the structure of the spatial pattern of synaptic potentiations. Densely clustered patterns will be substantially diminished, whereas sparse, spatially segregated ones will persist. The basis for this distinction is our finding that synapses flanking a potentiated synapse can balance its potentiation through distance-dependent suppression of their weights, somewhat similar to the lateral long-term depression found to accompany LTP in the short timescale (Royer and Pare, 2003), albeit over much shorter distances. The more spatially segregated a synaptic potentiation is, the more balancing suppression by other nearby synapses will be available for maintaining its potentiation. This implies that $l$-HSP can detect, and leave untouched, meaningful and relatively rare events, with a low probability of occurrence (low pLTP), worth being maintained.

This would seemingly be in opposition to the experimental finding that LTP elicited at a large group of synapses on a subtree of a CA1 neuron may spread out beyond the region of induction (Engert and Bonhoeffer, 1997). However, it is important to bear in mind that the LTP timescale is fast, whereas the homeostatic suppression of neighboring synapses predicted by our study is several orders of magnitude slower, so that these are two distinct phenomena. In addition, more recent experiments have demonstrated that LTP can be elicited at individual dendritic spines (Matsuzaki et al., 2004; Bagal et al., 2005), suggesting that mechanisms for the induction of very spatially segregated synaptic 
potentiations exit in the neuron. Importantly, we have shown that, under $l$-HSP, the dendritic tree with its many partially electrically decoupled branches (compartments) provides an ideal substrate for the endurance of a relatively high percentage of potentiated/depressed synapses.

Using the SIE measure for synaptic efficacy (London et al., 2002), we demonstrated that spatially sparse synaptic input will have a greater impact on the output of a neuron than a dense pattern. This is in agreement with recent theoretical work showing that an encoding strategy involving sparse synaptic potentiations within each dendritic subunit leads to optimal long-term information storage (B. Mel, personal communication). It is interesting that the predicted $l$-HSP selection rule, which is based on the large timescale local level of activity, happens to prefer such patterns of potentiation that are globally favorable at the short timescale.

With the advances in neurotransmitter uncaging techniques (Polsky et al., 2004; Shoham et al., 2005), an experiment could be designed in which, instead of the overall perturbation of the activity of the neuron (as is usually the case in HSP experiments), different spatial patterns of potentiation are induced (e.g., by an uncaging protocol) and the residual potentiation of identified synapses is measured several hours or days after LTP induction. In this way, our predictions regarding the dependence of the fate of LTP on the spatial configuration of synaptic potentiations can be directly tested. Interestingly, it has been shown recently that, in cerebellar Purkinje cells, metabotropic glutamate receptor subunit 1 receptors can detect and suppress spatially clustered patterns of synaptic activations but spare disperse ones through endocannabinoid-mediated plasticity (Marcaggi and Attwell, 2005). This presents an example for the existence of selectivity of spatial patterns of synaptic activation in dendrites.

\section{References}

Abarbanel HD, Gibb L, Huerta R, Rabinovich MI (2003) Biophysical model of synaptic plasticity dynamics. Biol Cybern 89:214-226.

Abbott LF, Nelson SB (2000) Synaptic plasticity: taming the beast. Nat Neurosci [Suppl] 3:1178-1183.

Abraham WC, Bear MF (1996) Metaplasticity: the plasticity of synaptic plasticity. Trends Neurosci 19:126-130.

Ariav G, Polsky A, Schiller J (2003) Submillisecond precision of the inputoutput transformation function mediated by fast sodium dendritic spikes in basal dendrites of CA1 pyramidal neurons. J Neurosci 23:7750-7758.

Bagal AA, Kao JP, Tang CM, Thompson SM (2005) Long-term potentiation of exogenous glutamate responses at single dendritic spines. Proc Natl Acad Sci USA 102:14434-14439.

Bienenstock EL, Cooper LN, Munro PW (1982) Theory for the development of neuron selectivity: orientation specificity and binocular interaction in visual cortex. J Neurosci 2:32-48.

Bliss TVP, Collingridge GL, Morris RGM, eds (2004) Long-term potentiation: enhancing neuroscience for 30 years. Oxford: Oxford UP.

Burrone J, Murthy VN (2003) Synaptic gain control and homeostasis. Curr Opin Neurobiol 13:560-567.

Burrone J, O’Byrne M, Murthy VN (2002) Multiple forms of synaptic plasticity triggered by selective suppression of activity in individual neurons. Nature 420:414-418.

Davis GW, Bezprozvanny I (2001) Maintaining the stability of neural function: a homeostatic hypothesis. Annu Rev Physiol 63:847-869.

Eilers J, Augustine GJ, Konnerth A (1995) Subthreshold synaptic $\mathrm{Ca}^{2+}$ signalling in fine dendrites and spines of cerebellar Purkinje neurons. Nature 373:155-158.

Engert F, Bonhoeffer T (1997) Synapse specificity of long-term potentiation breaks down at short distances. Nature 388:279-284.

Froemke RC, Poo MM, Dan Y (2005) Spike-timing-dependent synaptic plasticity depends on dendritic location. Nature 434:221-225.

Goldberg J, Holthoff K, Yuste R (2002) A problem with Hebb and local spikes. Trends Neurosci 25:433-435.
Golding NL, Spruston N (1998) Dendritic sodium spikes are variable triggers of axonal action potentials in hippocampal CA1 pyramidal neurons. Neuron 21:1189-1200.

Golding NL, Kath WL, Spruston N (2001) Dichotomy of action-potential backpropagation in CA1 pyramidal neuron dendrites. J Neurophysiol 86:2998-3010.

Johnston D, Hoffman DA, Colbert CM, Magee JC (1999) Regulation of back-propagating action potentials in hippocampal neurons. Curr Opin Neurobiol 9:288-292.

Ju W, Morishita W, Tsui J, Gaietta G, Deerinck TJ, Adams SR, Garner CC, Tsien RY, Ellisman MH, Malenka RC (2004) Activity-dependent regulation of dendritic synthesis and trafficking of AMPA receptors. Nat Neurosci 7:244-253.

Kempter R, Gerstner W, van Hemmen JL (2001) Intrinsic stabilization of output rates by spike-based Hebbian learning. Neural Comput 13:2709-2741.

Koch C, Bernander O, Douglas RJ (1995) Do neurons have a voltage or a current threshold for action potential initiation? J Comput Neurosci 2:63-82.

Leslie KR, Nelson SB, Turrigiano GG (2001) Postsynaptic depolarization scales quantal amplitude in cortical pyramidal neurons. J Neurosci 21:RC170(1-6).

Lisman J, Spruston N (2005) Postsynaptic depolarization requirements for LTP and LTD: a critique of spike timing-dependent plasticity. Nat Neurosci 8:839-841.

Lissin DV, Gomperts SN, Carroll RC, Christine CW, Kalman D, Kitamura M, Hardy S, Nicoll RA, Malenka RC, von Zastrow M (1998) Activity differentially regulates the surface expression of synaptic AMPA and NMDA glutamate receptors. Proc Natl Acad Sci USA 95:7097-7102.

London M, Schreibman A, Hausser M, Larkum ME, Segev I (2002) The information efficacy of a synapse. Nat Neurosci 5:332-340.

Magee JC, Cook EP (2000) Somatic EPSP amplitude is independent of synapse location in hippocampal pyramidal neurons. Nat Neurosci 3:895-903.

Marcaggi P, Attwell D (2005) Endocannabinoid signaling depends on the spatial pattern of synapse activation. Nat Neurosci 8:776-781.

Matsuzaki M, Honkura N, Ellis-Davies GC, Kasai H (2004) Structural basis of long-term potentiation in single dendritic spines. Nature 429:761-766.

Migliore M (1996) Modeling the attenuation and failure of action potentials in the dendrites of hippocampal neurons. Biophys J 71:2394-2403.

Migliore M, Hoffman DA, Magee JC, Johnston D (1999) Role of an A-type $\mathrm{K}^{+}$conductance in the back-propagation of action potentials in the dendrites of hippocampal pyramidal neurons. J Comput Neurosci 7:5-15.

Miller KD (1996) Synaptic economics: competition and cooperation in synaptic plasticity. Neuron 17:371-374.

Moulder KL, Cormier RJ, Shute AA, Zorumski CF, Mennerick S (2003) Homeostatic effects of depolarization on $\mathrm{Ca}^{2+}$ influx, synaptic signaling, and survival. J Neurosci 23:1825-1831.

O’Brien RJ, Kamboj S, Ehlers MD, Rosen KR, Fischbach GD, Huganir RL (1998) Activity-dependent modulation of synaptic AMPA receptor accumulation. Neuron 21:1067-1078.

Paradis S, Sweeney ST, Davis GW (2001) Homeostatic control of presynaptic release is triggered by postsynaptic membrane depolarization. Neuron 30:737-749.

Pawlak V, Schupp BJ, Single FN, Seeburg PH, Kohr G (2005) Impaired synaptic scaling in mouse hippocampal neurones expressing NMDA receptors with reduced calcium permeability. J Physiol (Lond) 562:771-783.

Polsky A, Mel BW, Schiller J (2004) Computational subunits in thin dendrites of pyramidal cells. Nat Neurosci 7:621-627.

Rabinowitch I, Segev I (2006) The interplay between homeostatic synaptic plasticity and functional dendritic compartments. J Neurophysiol 96:276-283.

Rall W (1959) Branching dendritic trees and motoneuron membrane resistivity. Exp Neurol 1:491-527.

Rao A, Craig AM (1997) Activity regulates the synaptic localization of the NMDA receptor in hippocampal neurons. Neuron 19:801-812.

Reid CA, Dixon DB, Takahashi M, Bliss TV, Fine A (2004) Optical quantal analysis indicates that long-term potentiation at single hippocampal mossy fiber synapses is expressed through increased release probability, recruitment of new release sites, and activation of silent synapses. J Neurosci 24:3618-3626.

Ross WN, Nakamura T, Watanabe S, Larkum M, Lasser-Ross N (2005) Syn- 
aptically activated $\mathrm{Ca}^{2+}$ release from internal stores in CNS neurons. Cell Mol Neurobiol 25:283-295.

Royer S, Pare D (2003) Conservation of total synaptic weight through balanced synaptic depression and potentiation. Nature 422:518-522.

Rumsey CC, Abbott LF (2004) Equalization of synaptic efficacy by activityand timing-dependent synaptic plasticity. J Neurophysiol 91:2273-2280.

Shoham S, O'Connor DH, Sarkisov DV, Wang SS (2005) Rapid neurotransmitter uncaging in spatially defined patterns. Nat Methods 2:837-843.

Siegel M, Marder E, Abbott LF (1994) Activity-dependent current distributions in model neurons. Proc Natl Acad Sci USA 91:11308-11312.

Sjostrom PJ, Turrigiano GG, Nelson SB (2001) Rate, timing, and cooperativity jointly determine cortical synaptic plasticity. Neuron 32:1149-1164.

Song S, Miller KD, Abbott LF (2000) Competitive Hebbian learning through spike-timing-dependent synaptic plasticity. Nat Neurosci 3:919-926.

Stuart G, Spruston N, Sakmann B, Hausser M (1997) Action potential initiation and backpropagation in neurons of the mammalian CNS. Trends Neurosci 20:125-131.

Sutton MA, Wall NR, Aakalu GN, Schuman EM (2004) Regulation of dendritic protein synthesis by miniature synaptic events. Science 304:1979-1983.

Sutton MA, Ito HT, Cressy P, Kempf C, Woo JC, Schuman EM (2006) Miniature neurotransmission stabilizes synaptic function via tonic suppression of local dendritic protein synthesis. Cell 125:785-799.

Thiagarajan TC, Piedras-Renteria ES, Tsien RW (2002) alpha- and beta-
CaMKII. Inverse regulation by neuronal activity and opposing effects on synaptic strength. Neuron 36:1103-1114.

Thiagarajan TC, Lindskog M, Tsien RW (2005) Adaptation to synaptic inactivity in hippocampal neurons. Neuron 47:725-737.

Toyoizumi T, Pfister JP, Aihara K, Gerstner W (2005) Generalized Bienenstock-Cooper-Munro rule for spiking neurons that maximizes information transmission. Proc Natl Acad Sci USA 102:5239-5244.

Turrigiano GG, Nelson SB (2000) Hebb and homeostasis in neuronal plasticity. Curr Opin Neurobiol 10:358-364.

Turrigiano GG, Nelson SB (2004) Homeostatic plasticity in the developing nervous system. Nat Rev Neurosci 5:97-107.

Turrigiano GG, Leslie KR, Desai NS, Rutherford LC, Nelson SB (1998) Activity-dependent scaling of quantal amplitude in neocortical neurons. Nature 391:892-896.

van Rossum MC, Bi GQ, Turrigiano GG (2000) Stable Hebbian learning from spike timing-dependent plasticity. J Neurosci 20:8812-8821.

Yeung LC, Shouval HZ, Blais BS, Cooper LN (2004) Synaptic homeostasis and input selectivity follow from a calcium-dependent plasticity model. Proc Natl Acad Sci USA 101:14943-14948.

Zador A, Koch C (1994) Linearized models of calcium dynamics: formal equivalence to the cable equation. J Neurosci 14:4705-4715.

Zelles T, Boyd JD, Hardy AB, Delaney KR (2006) Branch-specific $\mathrm{Ca}^{2+}$ influx from $\mathrm{Na}^{+}$-dependent dendritic spikes in olfactory granule cells. J Neurosci 26:30-40. 